\title{
Site Planning of a Newly Installed LINAC at BAEC, Bangladesh
}

\author{
Rajada Khatun ${ }^{1, *}$, Masud Rana², Monjur Ahasan', Shirin Akter', Fakhar Uddin", \\ Ashrafun Nahar Monika ${ }^{1}$, Md Ohiduzzaman ${ }^{3}$ \\ ${ }^{1}$ Medical Physics Research Group, Atomic Energy Centre, Bangladesh \\ ${ }^{2}$ Department of Oncology, Bangabondhu Sheikh Mujib Medical University(BSMMU), Bangladesh \\ ${ }^{3}$ Department of Biomedical Physics and Technology, University of Dhaka, Bangladesh
}

Copyright $@ 2017$ by authors, all rights reserved. Authors agree that this article remains permanently open access under the terms of the Creative Commons Attribution License 4.0 International License

\begin{abstract}
Radiotherapy is a multidisciplinary area which uses complex equipment and radiation sources for treatment. Radiotherapy programme is mainly run on site planning, constructing and staffing the radiotherapy facility by skilled people. Medical Physicists play a vital role in radiotherapy such as treatment plan verification, machine calibration, patient positioning etc. There is a lack of qualified medical physicist in Bangladesh. Considering this situation, Bangladesh Government has initiated an "Institute of Nuclear Medical Physics (INMP)" under the umbrella of Bangladesh Atomic Energy Commission. Linear Accelerator (LINAC) is the major equipment of this Institute. The main motto of this Institute is to provide educational opportunity and, training of the medical physics professionals as well as patient service. The location and siting of a radiotherapy facility within the hospital environment requires careful consideration due to the role of radiation oncology in multidisciplinary cancer management, including the needs for diagnosis, corresponding medical appointment and long term follow-up of patients. The specialized bunkers (shielded rooms) for housing the treatment equipment is technically an engineering challenge and needs professional oversight to ensure long term structural integrity. A standard design is imperative to provide future requirements and advances in technology. This study provides a basis for site planning of a newly installed radiotherapy facility (LINAC) construction.
\end{abstract}

Keywords LINAC, Bunker, TPS, Maze, Primary Barrier, Secondary Barrier

\section{Introduction}

The incidence of cancer is increasing day by day. According to recent estimates of the International Agency for Research on Cancer (IARC) and the World Health
Organization (WHO), approximately ten million new cases of cancer are being detected per year from worldwide, with slightly more than half of the cases occurring in developing countries like Bangladesh. In the year 2015 this number is about 15 million, of which two thirds will occur in developing countries. About half of all cancer patients receive radiotherapy, either as a part of their primary treatment or in connection with recurrences or palliative treatment.

Bangladesh is a developing country and is now facing many challenges, especially in the health sector. Cancer management is a priority due to the current trend of cancer diseases in this region. An accurate number of cancer patients are currently unavailable, but it is estimated that there are around 1.3 to 1.5 million cancer patients in the country with 150 million people, and about 0.2 million new cases are being added in every year. Around 150,000 cancer patients die annually [1]. Cancer is predicted to be an important cause of morbidity and mortality in Bangladesh in the future. According to the Bangladesh Bureau of Statistics, cancer is the sixth leading cause of death. IARC has estimated cancer-related death rates in Bangladesh to 7.5\% in 2005 and $13 \%$ in 2030 [2].

The first teletherapy (cobalt 60) machine was installed at a private hospital named Kumudini Hospital at Mirzapur in 1953. The second cobalt 60 machine was installed at Dhaka Medical College Hospital (DMCH) in 1959. The third radiation therapy center with deep X-ray machine was installed at Chittagong Medical College in 1964. The only tertiary-level cancer hospital of the country, NICRH, was founded privately in 1981 by Rotary Club of Dhaka as Rotary Cancer Detection Centre. It was handed over to the government in 1986. It started its indoor activities as a public hospital with 50 beds in 1992. The cobalt 60 machine was installed at this center in 1995 [3]. Research facilities are available at tertiary care centers and a few multi- country collaborative research activities are ongoing. Bangladesh has 
a unique National Cancer Control Strategy and Plan of Action 2009-15 formulated with the assistance of WHO with an objective to reduce the morbidity and mortality of cancer through primary prevention, early detection, and effective diagnosis and treatment. The strategy includes early detection and minimal therapy at the periphery and early detection and comprehensive multidisciplinary protocol-based therapy. As LINAC is a very useful tool in the treatment and management of cancers, many of these patients go to neighboring countries to get better treatment.

There was a long cherished desire that a well-equipped Medical Physics Institute would be established in Bangladesh for quality cancer care and management using medical physics technologies. In developed countries, a medical physicist needs to be trained up from proper Institute. The scope of medical physics education in Bangladesh is rare. Hence, an ADP project entitled as "Establishment of Institute of Nuclear Medical Physics" has been taken at Atomic Energy Research Establishment, Savar, Dhaka. This Institute would work to build up qualified medical physics professionals and technologists to deliver services to the patients. Consequently, international standard cancer diagnosis and treatment facilities would be developed through medical physics technologies.

The high cost of treatment, need of adequate radiation facilities, shortage of trained manpower, and lack of awareness within the community are the main challenges preventing cancer in Bangladesh. Inadequacy of trained medical physicist is a significant barrier in the developing of radiation treatment facilities in the country. As LINAC is a highly sophisticated machine, it is not so easy to be installed like other equipment. And it is very important to set up the facility with careful/perfect mode. So, to setup/site planning of a newly installed LINAC, we have to consider various aspects such as location, socio-economic status, shielding, system components, regulatory requirements, etc. The present study will help understanding to obtain a brief guideline for the purchaser/user of how to set up a new radiotherapy facility like LINAC.

\section{System Components and Site Planning Considerations}

Location of the LINAC is very important. Besides this, the LINAC must contain control console room, a simulator room, a treatment planning room, a mold room, a treatment room (bunker) and waiting areas. These rooms must be designed according to the manufacturer. Also, power, air conditioning, monitoring ports and emergency system should be included.

\subsection{Location}

Radiotherapy departments are usually located on the periphery of the hospital complex to avoid radiation protection problems arising from therapy rooms being adjacent to high occupancy areas. As pointed out in NCRP 49 [4], operational efficiency, initial cost, as well as provision for future expansion and/ or increased workload, should be considered when locating a therapy installation. For rooms below ground level, the reduction in shielding costs for floors and outside walls should be weighed against the expense of excavation, watertight sealing and of providing access. For rooms on or above ground level, the outside walls always require shielding; and additional structural support may be required for heavy equipment and for the additional weight of the shielding barriers. Our establishment will be setup above ground level. The schematic diagram of the facility is shown in Figure.1 and Figure.2.

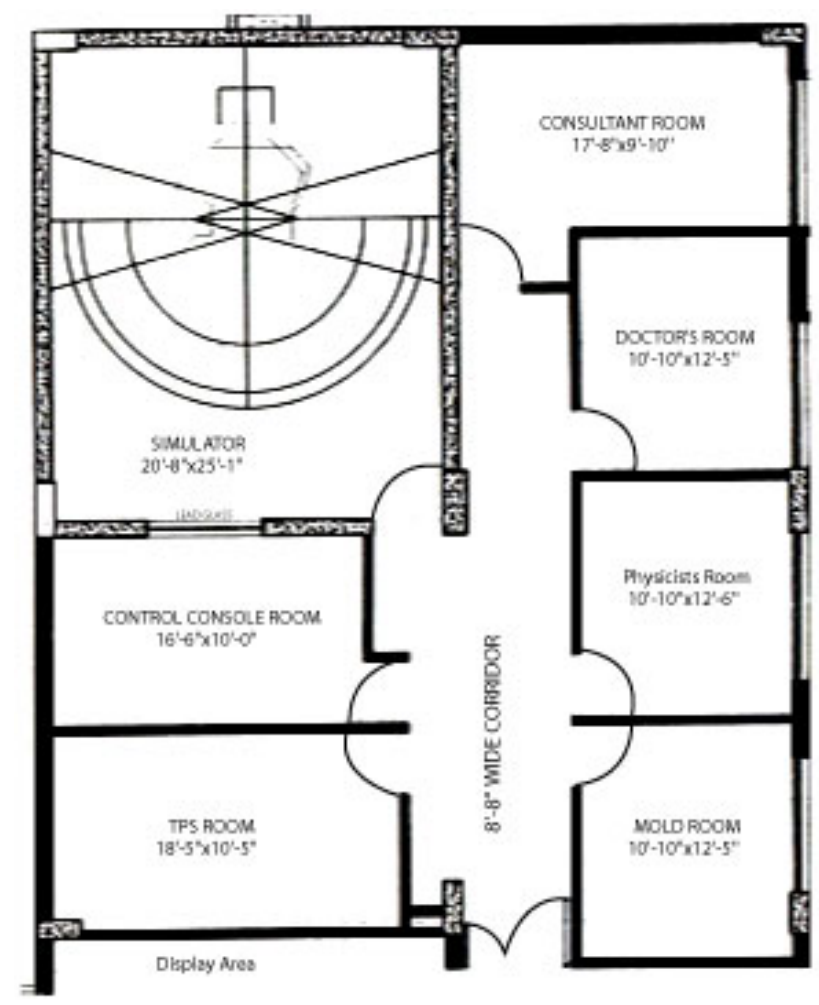

Figure 1. Typical Site Plan of the LINAC 


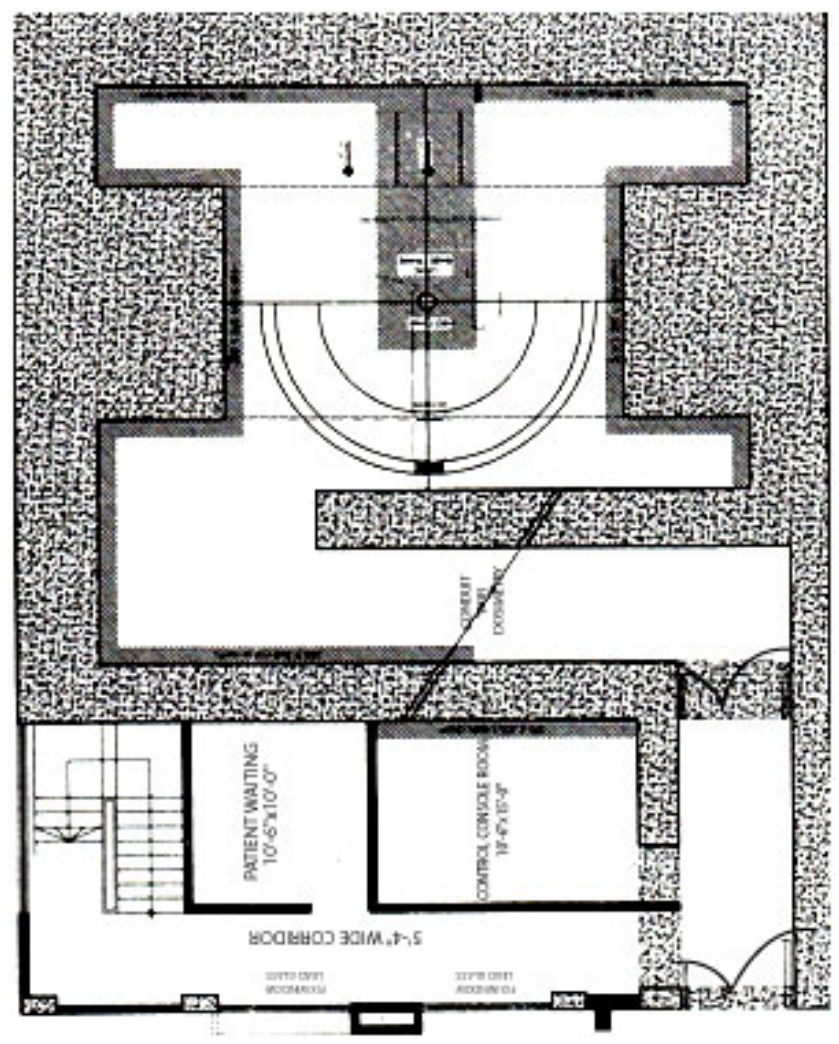

Figure 2. Bunker Design of the LINAC

\subsection{Treatment Room/Bunker}

The bunker will house a dual photon energy (6 MV and 15 $\mathrm{MV}$ ) and 5 electron energies (6, 9, 12, 15 and $18 \mathrm{MeV}$ ) accelerator. Bunker usually consists of an entrance (which may be a neutron door), a maze, a primary and a secondary barrier. The primary barrier is designed to attenuate the primary radiation beam that is produced by the linear accelerator. The thickness of the barrier depends on the most penetrating energy produced by the linear accelerator. For 15 $\mathrm{MV}$, a barrier thickness of $260 \mathrm{~cm}$ concrete is sufficient; this thickness can be reduced by using more attenuating materials (e.g. dense of concrete or materials). The secondary barrier is designed to attenuate scattered radiation from the primary beam as well as leakage radiation from the treatment head. Leakage radiation is typically of higher energy than scattered radiation, and therefore the thickness of the secondary barrier is typically determined by the leakage radiation. The secondary barrier thickness is typically half of the primary barrier thickness - e.g. $130 \mathrm{~cm}$ concrete for $15 \mathrm{MV}$ beams [5]. The maze typically designed to prevent the scattered photons from exiting the bunker directly and reduces the amount of radiation that escapes from the bunker by a significant amount. A maze that is sufficient to attenuate scattered photons may not be sufficient for scattered neutrons, which may be scattered multiple times. This may be overcome through the use of a larger maze (which causes neutrons to interact an increased number of times before reaching the exit) or through the use of a neutron door [6]. In the present establishment the primary shielding for $15 \mathrm{MV}$ LINAC is $11^{\prime} 10^{\prime \prime}$, secondary shielding is $4^{\prime} 7^{\prime \prime}$ and the length of the maze is $27^{\prime}$.

Technical Specification of the LINAC:

Model: Clinac iX Accelerator (VARIAN Medical System, Palo Alto, California, USA)

Photon Energy: $6 \mathrm{MV}, 15 \mathrm{MV}$

Electron Energy: 6, 9, 12, 15, $18 \mathrm{MeV}$

Dose Rate: 600 MU for Photon beam

Field Size: $0.5 \times 0.5-40$ x $40 \mathrm{~cm}^{2}$

Multi Leaf Collimator: 120 leafs (60 pairs) covering a field size of $40 \times 40 \mathrm{~cm}$.

\subsection{Control Console Room}

The treatment control room is where operators control the machine. This area should be close to the entrance to the treatment bunker so that the operators can view the entrance area. The control room should be sufficiently large to accommodate the treatment unit control console and associated equipment. There may be computer terminals for record and verification, electronic portal imaging, hospital information system and dosimetry equipment, as well as closed circuit TV monitors for patient observation. There should be clear access to any dosimetry ducts.

\subsection{Treatment Planning Room}

The Treatment Planning Room can be located anywhere, and configured in many ways, depending upon the site layout and desire of the customer. Typically, the Treatment Planning Room should include enough workspace for two or more workstations and a desktop color laser printer. It should be located within 328 feet (100 meters) network cable run length to the Equipment Room. Space is also required for light boxes. A high intensity light is for viewing CT scans and plane X-ray films. This will facilitate better Information Technology (IT) connection speeds, as it will allow direct connection between the workstations located in this room and the LINAC internal network. This must be situated close to simulator room but need not to be adjacent.

\subsection{Simulator Room}

CT simulators are used to plan treatment. The room must be shielded properly. It should be enough large to accommodate simulator machine. The patient positioning lasers to the wall through the isocentre should be included in the plans. Dimming lights should be included in the room. The room requires a sink. If immobile devices are to be installed in the room, cabinet space to store supplies for their fabrication will be required.

\subsection{Mold Room}

Mold room space should be provided in a radiotherapy facility. It is necessary to fabricate custom designed blocks and compensators. A block cutter and counter-top workspace 
for pouring and mounting the blocks and tools space should be provided. Storage space for supplies of styrofoam, trays and shielding material for custom blocking is necessary.

\section{Radiation Shielding}

\subsection{Shielding for LINAC}

Patient safety is very important and is assured in several ways. Safety of the staff operating the linear accelerator is also important. The linear accelerator sits in a room with lead and concrete walls so that the high-energy $\mathrm{X}$-rays are shielded and no one outside of the room is exposed to the $\mathrm{X}$-rays. Concrete barriers designed for high $\mathrm{X}$-ray shielding are sufficient for protection against neutrons [7]. Typically, integrated dose rate limits for radiation therapy facility barrier design is $20 \mu \mathrm{Sv} /$ week for uncontrolled areas and $100 \mu \mathrm{Sv} /$ week for controlled areas [8]. Protection is required against three types of radiation:

- 1 . Primary radiation (from the patient)

- 2. Scattered radiation (from the patient)

- $\quad 3$. Leakage radiation (from the head)

\subsection{Leakage Rates}

The LINAC is shielded to limit the leakage to an average of about $0.03 \%$ of the primary beam. Maximum value of leakage rate at distance of 1 meter from the beam path is limited to $0.1 \%$ of the primary beam dose rate at $80 \mathrm{~cm}$ from the x-ray target (area $<10 \mathrm{~cm}$ x $10 \mathrm{~cm}$ ). Both leakage and TVL values are smaller around the back of the LINAC. Leakage around the rest of the LINAC is $29.2 \pm 0.4 \mathrm{~cm}$ of concrete. Typically, only $50 \%$ of the leakage dose value is involved of radiation that is of sufficient energy to penetrate more than one TVL of concrete.

\subsection{Regulatory Requirements}

The customer is responsible for obtaining all local, state and national permits and requirements associated with site planning, shielding, site preparation, construction, system installation and system maintenance. In Bangladesh, Bangladesh Atomic Energy Regulatory Authority (BAERA) is responsible for all regulatory aspects. A full radiation survey including the photon and neutron leakage measurements will still have to be completed to comply with regulatory requirements after a full calibration. The preliminary survey is needed to assure the safety of individuals during the acceptance testing and commissioning.

\section{Electrical and Environmental Requirements}

\subsection{Electrical}

It is recommended to supply $480 \mathrm{VAC}$, 3-phase, 100 Amps, $55 \mathrm{kVA}$ power to the Main Power Disconnect. However, LINAC will accept input power in the range of 200 VAC through 480 VAC. For any input voltages at 240 VAC and below, 150 Amps is required. The Main Power Disconnect typically needs a 36 inch square (914 millimeter square) exclusionary area directly in front of it for regulatory requirements. It should be located next to the door of the Equipment Room, with the Junction Box located between the Main Power Disconnect and the door. The customer is responsible for the Disconnect box, fuses and all conduits and wiring from the original power source to the box. A grounding lug is to be supplied by the electrical contractor with the following specifications: A 4 gauge lug terminating to grounded building steel or earth within the main power disconnect. The Main Disconnect can be located on an outside wall of the Equipment Room, as long as it remains within the cable limitations of the PDU. An online UPS must be provided to power the Treatment Delivery, Data Server, LCD monitor and networking devices in the event of a power failure to reduce risk of lost or damage to data.

\subsection{Environmental}

The Treatment Room should be kept between $50^{\circ} \mathrm{F}$ and $85^{\circ} \mathrm{F}\left(10^{\circ} \mathrm{C}\right.$ and $\left.30^{\circ} \mathrm{C}\right)$, twenty-four hours per day, seven days per week, with a range of 30 to $70 \%$ relative humidity. The customer may choose to incorporate noise reduction in the equipment room. The noise level is measured to be $90 \mathrm{~dB}$. The customer is responsible for any kind of external vibration that may affect the equipment.

\section{Other System Implementation Considerations}

\subsection{Patient Positioning Laser}

The system will have three lasers installed on the walls of the treatment room. An optional (fourth laser) laser is attached on the ceiling or I-section girder of the treatment room. Laser specifications are as follows:

- Wall laser $(\times 2)$

- Ceiling laser (optional)

- $\quad$ Sagittal laser

\subsection{Warning Sign and Light}

The BSS [9] requires registrants and licensees to display a warning symbol, such as that recommended by the International Organization for Standardization (ISO) at access points and other appropriate locations within controlled areas. It is recommended that an illuminated warning sign should be displayed at the entrance to the maze or treatment bunker as well as several inside the treatment bunker. It should be possible to see a warning sign from any position within the treatment bunker. These signs should be 
mounted at eye level (1650mm above finished floor level) and interlocked with the treatment control unit. The illuminated signs may have two or three stages. For a two stage sign, the first stage will be illuminated when there is power to the treatment unit, and the second stage will illuminate when the beam is turned on. For a three stage sign, stage one will be illuminated when there is power to the treatment unit, stage two will light when the treatment unit is programmed to deliver a radiation beam and stage three will illuminate when the beam is turned on. A warning sign indicate the nature of the hazard [10].

\subsection{Patient Observation and Monitoring}

The operator should be visually monitoring the patient during treatment with closed circuit TV. Two cameras are needed. The cameras should be located far away from the radiation source, and consistent with tele-zoom capabilities to minimize degradation of the image receptor by scatter radiation. There should also be provision for two-way audio-communication between the treatment control area and the room. A patient activated alarm may be required for patients unable to give an audible call.

\subsection{Quality Assurance and Commissioning Tools and Equipment}

Quality control of the linear accelerator is also very important. There are several systems built into the accelerator so that it will deliver exact dose that the radiation oncologist has prescribed. There are various acceptance tests /protocols to meet the requirements of IAEA/AAPM or other reputed Association [11]. The Medical Physicists are responsible to make specifications of all QA tools and equipment and to perform the entire acceptance tests of the LINAC machine at the installation and commissioning period. Report and documentation of all the performance parameters given by the manufacturer is also very important for further maintenance of the machine.

\section{Conclusions}

The necessity of radiotherapy facility/LINAC in Banglades is unquestionable. Number of cancer patient is increasing day by day and in future the need of radiotherapy will increase furthermore. We don't only need radiotherapy machine but also successful installation of the newly purchased LINAC to deliver quality treatment. It would require quality equipment, staff, radiologist, oncologist and other necessary elements to provide better services. Otherwise, the main goal will be void. Setting up such a sophisticated radiotherapy machine is not easy. As Bangladesh is a developing country, it is highly recommended to plan or design a radiotherapy centre in accordance with the given conditions before starting construction. Else, valuable money and time would be wasted, and patients won't be satisfied to the service provided by the LINAC. Hence, well planning of a LINAC is very important in perspective of Bangladesh. This study will provide basic information line on site planning/set up of a digital LINAC.

\section{REFERENCES}

[1] Hussain SM, Comprehensive update on cancer scenario of Bangladesh, South Asian J Cancer, 2(4):279-84, 2013.

[2] Hussain SA, Sullivan R., Cancer control in Bangladesh, Jpn J Clin Oncol., 43(12):1159-69, 2013.

[3] Abdullah Abu Zaher, Setting Up a Radiotherapy Centre, MSc Poject, Department of Physics, Jahangirnagar University, Savar, Dhaka, 2015.

[4] National Cancer Control Strategy and Plan of Action 2009-15. Directorate General of Health Services. Ministry of Health and Family Welfare, Bangladesh.

http://www.ban.searo.who.int/LinkFiles/Publication_Cancer_ Strategy.pdf.pdf., 2012.

[5] National Council on Radiation Protection and Measurements, Structural Shielding Design and Evaluation for Medical Use of X-rays and Gamma-rays of Energies up to $10 \mathrm{MeV}$, Rep. 49, NCRP, Washington, DC, 1976.

[6] IPEM Report No.75, The Design of Radiotherapy Treatment Room Facilities, IPEM, New York, 1997.

[7] The Physics of Radiation Therapy. F. M. Khan, 4th edition, p. 365.

[8] Cyber knife® M6 Series Site Planning Guide, Part number: 501035, 2013

[9] International Basic Safety Standards for Protection against Ionizing Radiation and for the Safety of Radiation Sources, Safety Series No.115, IAEA, Vienna, 1996.

[10] International Organization for Standardization, Basic Ionizing Radiation Symbol, ISO-1361, ISO, Geneva, 1975.

[11] IAEA Human Health Reports No.10, Radiotherapy Facilities: Master Planning and Concept Design Considerations, Vienna, 2014. 